\title{
Control of radio-frequency atmospheric pressure argon plasma characteristics by helium gas mixing
}

\author{
Se Youn Moon, Jewoo Han, ${ }^{\text {a) }}$ and W. Choe ${ }^{\text {b) }}$ \\ Department of Physics, Korea Advanced Institute of Science and Technology, 373-1 Guseong-dong, \\ Yuseong-gu, Daejeon 305-701, Korea
}

(Received 18 July 2005; accepted 29 November 2005; published online 12 January 2006)

\begin{abstract}
The control of plasma characteristics is one of the important issues in many atmospheric pressure plasma applications. In order to accomplish this control, a feasibility study was performed by investigating the role of helium gas in an argon glow plasma that were produced in ambient air by 13.56 MHz radio-frequency power. Optical emission spectroscopy was used to measure rotational temperature and emission spectra acquired between 300 and $840 \mathrm{~nm}$. Based on electrical and optical measurements, parameters such as gas temperature, breakdown voltage, power coupling efficiency, spatial uniformity of rotational temperature, and the sum of the emission intensity were controlled by varying the argon and helium gas mixing ratio. The addition of helium gas (from 0 to $10 \ell \mathrm{pm}$ ) to the argon flow (of $10 \ell \mathrm{pm}$ ) lowered the breakdown voltage (from 430 to $300 \mathrm{~V}_{\mathrm{pk}}$ ) and the rotational temperature (from 465 to $360 \mathrm{~K}$ ). However, an excessive addition of helium resulted in a reduction of the spatial uniformity and efficiency of power coupling. When the ratio of helium to argon flow was between 0.3 and 0.5 , a high spatial uniformity with a relatively low gas temperature and breakdown voltage was achieved. This suggests that mixing of the supply gas is a useful way of controlling the plasma characteristics that may be utilized for applications with specific required discharge conditions. (C) 2006 American Institute of Physics. [DOI: 10.1063/1.2161173]
\end{abstract}

\section{INTRODUCTION}

Atmospheric pressure plasmas have been recently utilized for various industrial applications, and it is expected that the low-pressure plasmas now routinely used in many sophisticated processes will be replaced by atmospheric pressure plasmas. In order for this to take place, large volume, high spatial uniformity, and discharge stability are some prerequisites to be satisfied for the wide use of atmospheric pressure plasmas. In particular, plasmas produced in ambient air are given special attention due to their simple setup. In many atmospheric pressure plasmas being utilized in various industrial processes, gases such as argon or helium are often introduced for assisting stable plasma generation and/or for other particular processes. In general, helium has lower breakdown voltage characteristics in the $>10$ Torr $\mathrm{cm}$ range of the Paschen curve $\mathrm{e}^{1-3}$ and a lower gas temperature ${ }^{4}$ than argon, which is consistent with our experimental results. The low breakdown voltage is beneficial because it helps by preventing arc production and system overloading. Low gas temperature is also important in some atmospheric pressure plasma sources for treating materials such as films, glasses, and biomaterials that are prone to thermal damage. However, argon has its own merits as well in that it is less expensive and it is relatively easier to produce large volume plasmas.

Based on the discharge characteristics with each type of gas mentioned above, we investigated how a mixture of

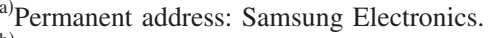

b) Author to whom correspondence should be addressed. Electronic mail: wchoe@kaist.ac.kr
}

argon and helium as the supply gas affects the overall characteristics of the plasma produced in ambient air in order to take advantage of the merits of both gases.

\section{EXPERIMENTAL SETUP}

Figure 1 shows a schematic illustration of the present experimental setup for which a detailed description is found elsewhere. ${ }^{5}$ A volumetric and stable glow plasma is generated with the help of a double structure which consists of two side-discharge gaps and a bottom-discharge gap. The dielectric plates covering the side electrodes limit the overcurrent that leads to arc and assist in the production of a stable, uniform glow plasma. The widths of the side-discharge gaps and the bottom-discharge gap were fixed at 1 and $3 \mathrm{~mm}$, respectively, for this work. The central electrode was powered by a $13.56 \mathrm{MHz}$ radio-frequency (rf) source through a matching network. The typical area of the main plasma at the bottom-discharge gap was $200 \mathrm{~mm}(x) \times 50 \mathrm{~mm}(y)$, where $x$ and $y$ directions are denoted in Fig. 1. The plasma was generated in ambient air with the supply gases introduced through a uniform array of holes in the side gaps. The electrical characteristics of the discharge were measured by a current probe (Tektronix TCP202) and a voltage probe (Tektronix P6015A) with a digital oscilloscope (Tektronix TDS3012). The visible spectrum and the emission intensity emitted from the discharge were acquired through a Chromex 250IS spectrometer with a charge-coupled device (CCD) detector. 


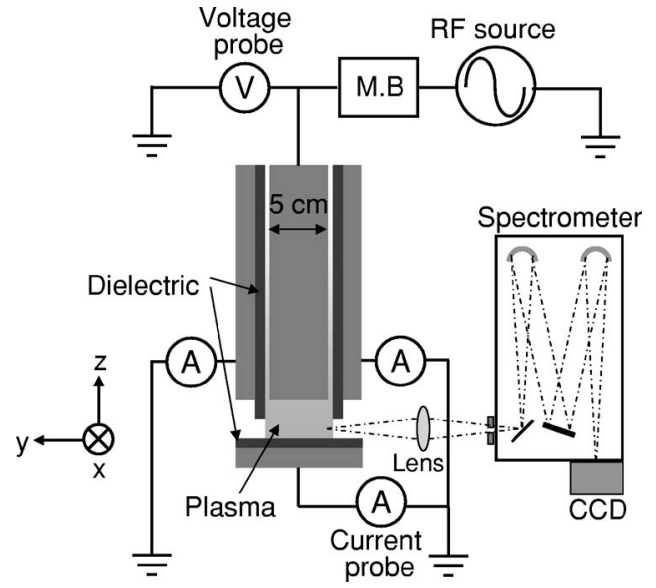

FIG. 1. Schematic illustration of the experimental setup.

\section{RESULTS}

Measurements showed that the breakdown voltage with the helium gas supply was $142 \mathrm{~V}_{\mathrm{pk}}$, which was only about $1 / 3$ of that with argon while other operation conditions were fixed. The lower breakdown voltage for helium than for argon at atmospheric pressure can be explained by the Penning effect of the helium metastables. ${ }^{6,7}$ Figure 2(a) shows the breakdown voltage $V_{b}$ as a function of the helium and argon mixing ratio, where the argon flow rate was fixed at $10 \ell \mathrm{pm}$ and the helium flow rate was varied from 0 to $10 \ell \mathrm{pm}$. Therefore, a He/Ar ratio of 1.0 corresponds to a case of $1: 1$ mixture of the helium and argon at $10 \ell \mathrm{pm}$ each. As the helium flow rate was increased, $V_{b}$ decreased from 425 to $300 \mathrm{~V}_{\mathrm{pk}}$ as expected, because of the dilution of the argon gas and the Penning ionization of argon atoms by he-

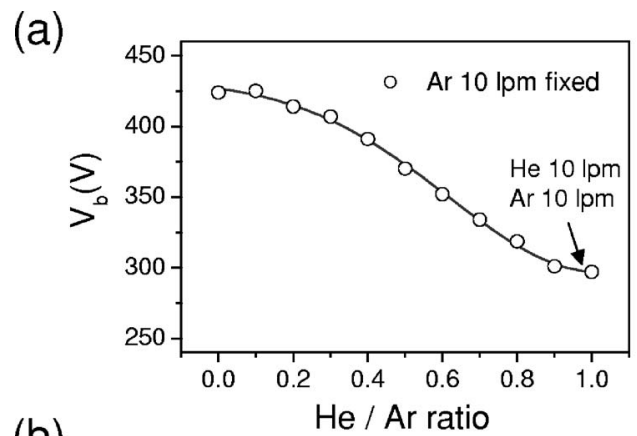

(b)

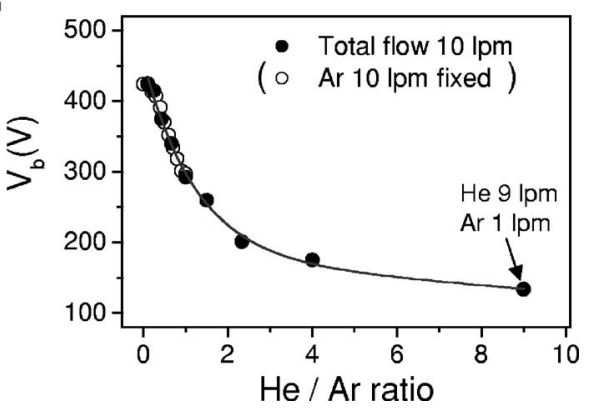

FIG. 2. Breakdown voltage at various argon-helium mixing ratios (a) with the argon flow rate fixed at $10 \mathrm{\ell pm}$ and the helium flow rate varying from 0 to10 $\ell \mathrm{pm}$ and (b) with the argon-helium total flow rate fixed at $10 \ell \mathrm{pm}$ and the flow rate of both gases varied. Results indicate that the breakdown voltage mainly depends on the mixing ratio rather than the total flow rate.

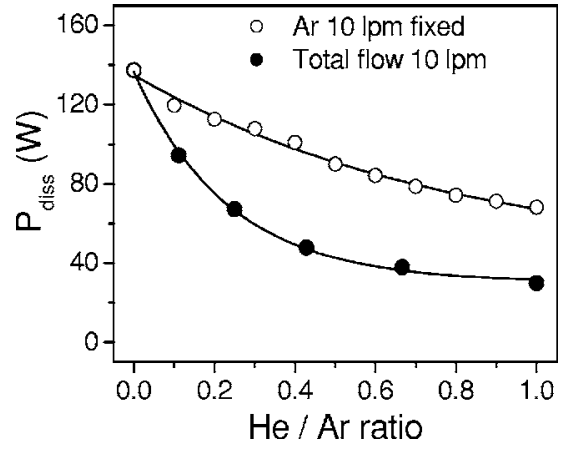

FIG. 3. Dissipated power at various He/Ar mixing ratios at $300 \mathrm{~W}$ rf power. The case of the constant argon flow rate (-O-) shows a better power coupling efficiency than the case of the constant total flow rate (-0-).

lium metastables. ${ }^{6}$ On the other hand, $V_{b}$ for the case of a fixed argon and helium total flow rate is depicted in Fig. 2(b) as closed circles. In this case, the argon flow rate was decreased from 10 to $1 \ell \mathrm{pm}$, and the helium flow rate was increased from 0 to $9 \ell \mathrm{pm}$, respectively, while the total flow rate was kept constant at $10 \ell \mathrm{pm}$. For example, the data point at the $\mathrm{He} / \mathrm{Ar}$ ratio of 9.0 corresponds to the case of helium and argon flow rates of 9 and $1 \ell \mathrm{pm}$, respectively. We plotted the data points (open circles) of Fig. 2(a) together with the closed circles in Fig. 2(b), where it is found that the open circles (cases with only the helium flow rate varying at a fixed argon flow rate) follow the curve of the closed circles (cases in which the total flow rate of the mixed gas was fixed). Figure 2(b) clearly indicates that $V_{b}$ depends mainly on the mixing ratio rather than the total flow rate.

Shown in Fig. 3 is the measured rf power dissipated in the plasma across the bottom-discharge gap for two different methods of varying the $\mathrm{He} / \mathrm{Ar}$ ratio of the gas mixture at $300 \mathrm{~W}$ of input rf power. Here, the dissipated power $P_{\text {diss }}$ during a period $T$ was defined as $P_{\text {diss }}=1 / T \int_{0}^{T} I(t) V(t) d t$. For both cases, $P_{\text {diss }}$ exponentially decreased as the amount of helium gas was increased. In addition, $P_{\text {diss }}$ was larger with the argon flow rate kept constant at $10 \ell \mathrm{pm}$ and the helium flow rate varied (-O-) than the case with the total flow rate fixed at $10 \ell \mathrm{pm}(-\mathbf{-})$. Since $V_{b}$ depends mainly on the mixing ratio rather than the total flow rate as noted above, the former case (-O-) is considered to be better because of the larger rf power dissipated into the plasma.

Figure 4(a) shows the discharge current $\left(I_{d}\right)$ and sustained voltage $\left(V_{d}\right)$ measured across the central-powered electrode and the grounded bottom conductor at $300 \mathrm{~W}$ and $10 \mathrm{\ell pm}$ argon flow with different helium flow rates. As shown in the figure, $V_{d}$ increased but $I_{d}$ decreased as the helium flow rate was increased for the $\mathrm{He} / \mathrm{Ar}$ ratio less than 0.4 . The plasma volume remained almost unchanged. In that range of the mixing ratio, the plasma resistance increases due to the $V_{d}$ increment and $\mathrm{I}_{d}$ decrement which resulted from the helium addition. This is consistent with the fact that the electron-neutral collision frequency in helium is higher than in argon at $1 \mathrm{~atm}^{8}$ At a He/Ar ratio larger than 0.4 , on the other hand, $V_{d}$ remained nearly constant, and $I_{d}$ continued to decrease. However, since the plasma area $S$ decreased in the 
(a)

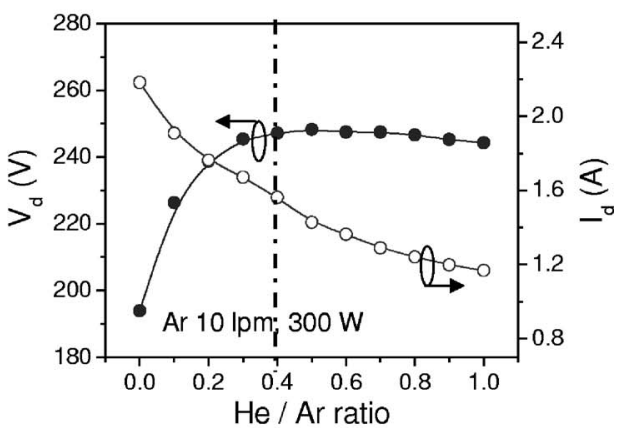

(b)

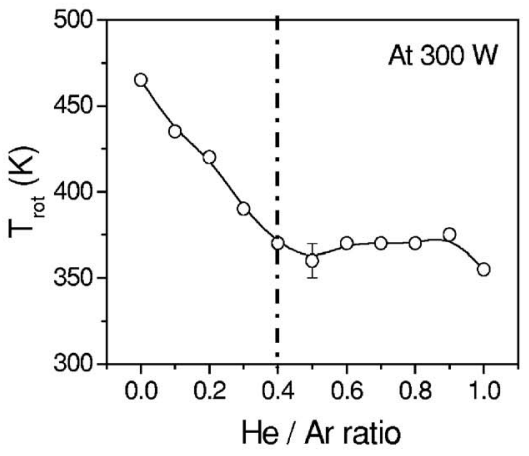

(c)

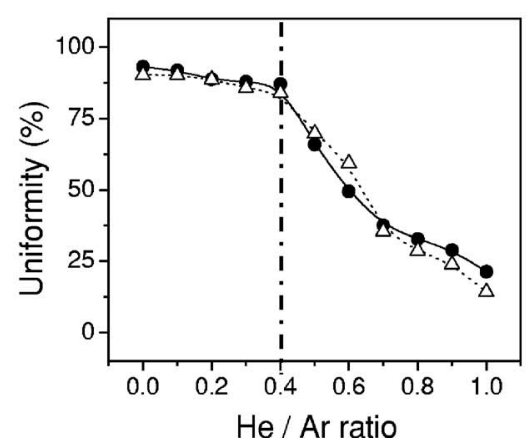

FIG. 4. (a) Discharge current and voltage, (b) rotational temperature $T_{\text {rot }}$, and (c) spatial uniformities of $T_{\text {rot }}\left(-\triangle_{-}\right)$and the sum of the emission intensity acquired by the CCD detector of the spectrometer in the spectral range between 300 and $840 \mathrm{~nm}(-\boldsymbol{-}-)$ at various mixing ratios. Helium addition reduces the discharge current $T_{\text {rot }}$ and the spatial uniformity.

bottom-discharge gap, the current density $I_{d} / S$ was nearly constant at $35.2 \mathrm{~mA} / \mathrm{cm}^{2}$.

The rotational temperature $T_{\text {rot }}$ was measured as depicted in Fig. 4(b) by analyzing the $\mathrm{OH}$ diatomic molecular spectrum emitted from the plasma due to the moisture in the ambient air and possibly due to feed gas impurities. ${ }^{9}$ As the amount of the helium addition increased, $T_{\text {rot }}$ linearly decreased until the gas mixing ratio became 0.4 . Thereafter, it remained in between 370 and $375 \mathrm{~K}$, which was due to the constant voltage and current density as noted above because $T_{\text {gas }}\left[\simeq T_{\text {rot }}(\right.$ Ref. 10) $]$ is known to be a function of the current density and voltage in normal glow discharges at atmospheric pressure. ${ }^{11}$ The spatial uniformity, defined as 1 $-(\max -\min ) /(\max +\min )$, of $T_{\text {rot }}(-\triangle-)$ and the sum of the emission intensity acquired by the CCD detector of the spectrometer in the spectral range between 300 and $840 \mathrm{~nm}(-\mathbf{-}-)$ were measured along the lengthwise direction to investigate how significantly the helium addition changed the spatial uniformity [Fig. 4(c)]. The optical measurements were per- (a)

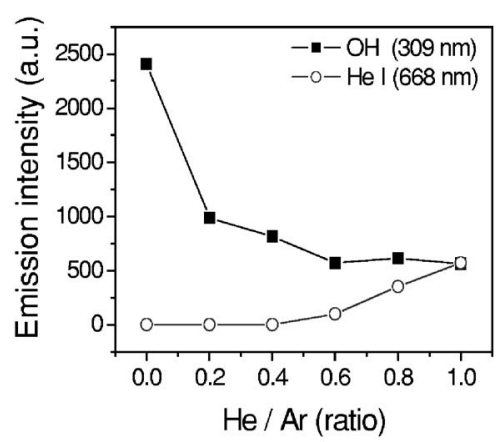

(b)

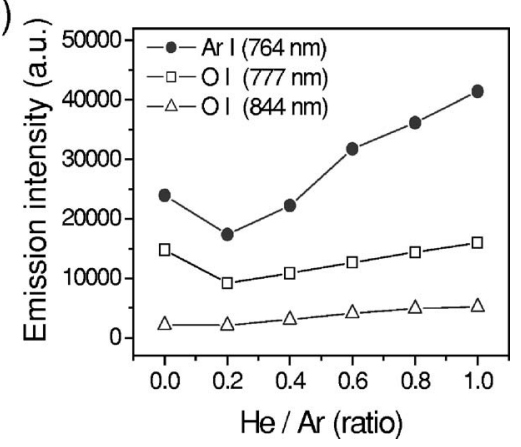

FIG. 5. Emission intensity from (a) excited helium atoms $(668 \mathrm{~nm})$ and $\mathrm{OH}$ molecules (309 nm) and (b) excited argon atoms (764 nm) and excited oxygen atoms (777 and $844 \mathrm{~nm}$ ) at various He/Ar mixing ratios.

formed at $1 \mathrm{~cm}$ intervals along the $x$ direction with a $1 \mathrm{~mm}$ resolution, which was determined by measuring the image size of a laser beam guided through an optical fiber of $1 \mathrm{~mm}$ core size. More than $90 \%$ uniformity was observed at a mixing ratio less than 0.4 , and it rapidly decreased at higher mixing ratios. This is a consequence of the decreased plasma volume, which resulted from the lower power coupling. Based on the measurement at $300 \mathrm{~W}$, a helium and argon mixing ratio of 0.4 seemed to be the optimum ratio, which offered a low discharge voltage and rotational temperature while keeping a relatively high spatial uniformity. Curves similar to Fig. 4(c) were observed at other input rf power levels and for different dielectrics such as glass at the same input rf power levels. In these cases, however, the mixing ratio at which the uniformity rapidly decreased shifted toward larger value. For example, the mixing ratio was 0.6 at $450 \mathrm{~W}$.

The optical emission measurement demonstrated that the emission intensity of the helium atoms $(668 \mathrm{~nm})$ increased as expected as more helium gas was added as shown in Fig. 5(a). However, the $\mathrm{OH}$ emission intensity, mainly produced by the collisions between argon atoms and the water impurity, decreased due to the argon dilution. ${ }^{12}$ On the other hand, it was observed that the excited argon atom intensity increased [Fig. 5(b)]. This is due to the Penning effect of helium metastables or the collisional energy transfer from helium to argon atoms, because the energy of the helium singlet metastable state $2 s^{1} S_{0}(20.62 \mathrm{eV})$ is larger than that of the argon excited state $(13.2 \mathrm{eV})$. In addition, the oxygen atom intensity (777 and $844 \mathrm{~nm}$ ) increased since oxygen atoms were also excited by the helium through electron-impact excitation. Greater concentration of oxygen atoms in the he- 

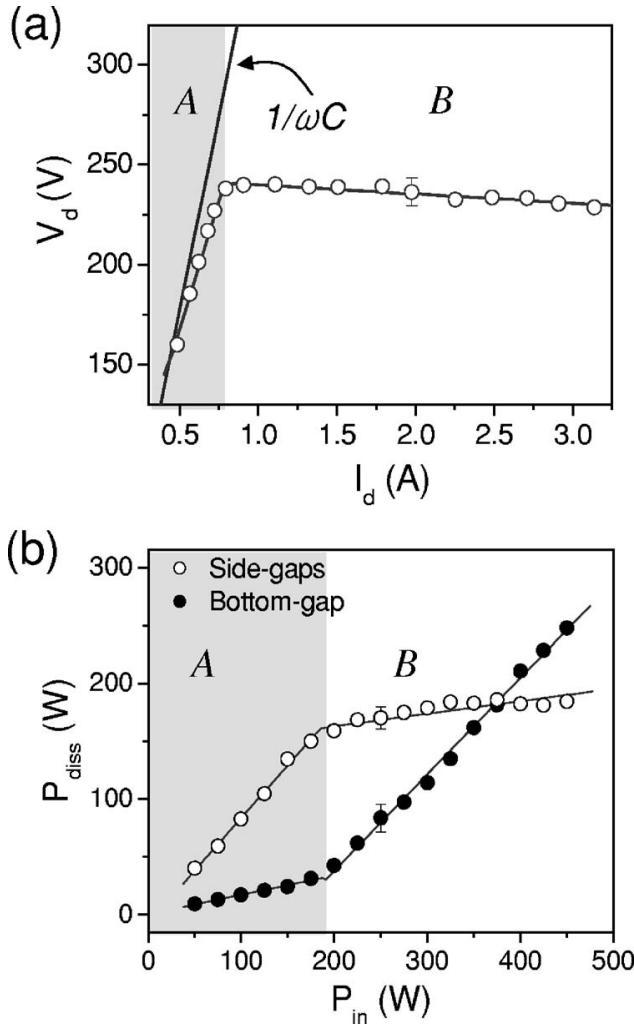

FIG. 6. (a) The measured current-voltage characteristic curve and (b) the dissipated power at the side gaps and the bottom-discharge gap.

lium plasma may also be due to the slower recombination of oxygen atoms with helium as a third body, instead of argon. $^{2,13}$ It suggests that a helium addition enhances the oxygen radical production.

Figure 6(a) represents the current versus voltage curve at the mixing ratio of 0.4 (i.e., $\mathrm{Ar}: \mathrm{He}=10 \ell \mathrm{pm}: 4 \ell \mathrm{pm}$ ) within our rf power supply limit (from 50 to $450 \mathrm{~W}$ ). In the low current region indicated by $A$, the discharge voltage linearly increased along with the current. The slope of the currentvoltage curve $d V_{d} / d I_{d}$ is similar to $1 / \omega C$, where $\omega(=2 \pi$ $\times 13.56 \mathrm{MHz})$ is the driving frequency and $C(=31 \mathrm{pF})$ is the capacitance of the bottom gap. It is because in the operation region $A$, only a small plasma, ejected from the sidedischarge gaps, existed in the space between the central electrode and the bottom conductor rather than a bulk plasma filling the space. The discrepancy between the $1 / \omega C$ line and the line connecting the open circles in the region $A$ is attributed to a small number of charged particles or excited atoms flowing out from the side-discharge gaps. On the other hand, it was observed that an active breakdown occurred at the bottom-discharge gap in the region indicated by $B$. By increasing the input power, the discharge voltage was nearly constant at about $235 \mathrm{~V}_{\mathrm{pk}}$ while the discharge current increased. However, the current density remained almost constant at $35.2 \mathrm{~mA} / \mathrm{cm}^{2}$ due to the plasma area increase, which is a typical characteristic of normal glow discharges. The fractions of the dissipated power in the side gaps and in the bottom gap is plotted in Fig. 6(b) as a function of the input power. It is noted that the reflected power was negligible compared to the forward rf power at all times due to the impedance matching circuit. Figure 6(b) shows that the dissipated power in the bottom increases with a higher slope than the dissipated power in the side gaps, indicating a more efficient input power consumption in the bottom gap once the active breakdown occurred at the bottom-discharge gap, as indicated in region $B$.

\section{SUMMARY}

We observed the changes in the plasma characteristics by changing the mixing ratio of helium to argon feed gas in a large area rf plasma source produced at atmospheric pressure in ambient air. From the electrical and optical measurements, it was demonstrated that the discharge characteristics and plasma-related parameters such as rotational temperature and spatial uniformity could be controlled through the argon and helium mixing ratio. Changing the mixing ratio by adding helium significantly reduced the discharge voltage and the rotational temperature. This may be very useful for some particular processes utilizing atmospheric pressure plasmas. However, the addition of too much helium gas reduced the power coupling efficiency and the spatial uniformity of $T_{\text {rot }}$ and the visible spectral emission intensity. It was also observed that there existed an optimum mixing ratio, depending on the input power level, at which a relatively low discharge voltage, a low gas temperature, a high uniformity, and a high power coupling efficiency were obtained. These experimental results allow us to expect that the characteristics of atmospheric pressure plasmas, produced in ambient air, can be effectively controlled by gas mixing for applications with specific requirements.

\section{ACKNOWLEDGMENTS}

This work was supported by KAIST. The authors thank PLASMART, Inc. for support.

${ }^{1}$ J. Park, I. Henins, H. W. Herrmann, and G. S. Selwyn, J. Appl. Phys. 89, 20 (2001).

${ }^{2}$ M. Moravej, X. Yang, G. R. Nowling, J. P. Chang, R. F. Hicks, and S. E. Babayan, J. Appl. Phys. 96, 7011 (2004).

${ }^{3}$ H. Okubo, S. Yuasa, K. Ota, N. Hayakawa, and M. Hikita, IEEE Trans. Electr. Insul. 4, 450 (1997).

${ }^{4}$ S. Wang, V. Schulz-von der Gathen and H. F. Döbele, Appl. Phys. Lett. 83, 3272 (2003)

${ }^{5}$ S. Y. Moon, B. K. Kang, and W. Choe, Appl. Phys. Lett. 84, 188 (2004).

${ }^{6}$ J. Park, I. Henins, H. W. Herrmann, and G. S. Selwyn, J. Appl. Phys. 89, 15 (2001).

${ }^{7}$ D. T. A. Blair, Electrical Breakdown of Gases, edited by J. M. Meek and J. D. Craggs (Wiley, Chichester, 1978).

${ }^{8}$ J. R. Roth, Industrial Plasma Engineering: Applications to Nonthermal Plasma Processing (IOP, Bristol, 2001), Vol. 2, Chap. 19.

${ }^{9}$ S. Y. Moon and W. Choe, Spectrochim. Acta, Part B 58, 249 (2003).

${ }^{10}$ S. Pellerin, J. M. Cormier, F. Richard, K. Musiol, and J. Chapelle, J. Phys. D 29, 726 (1996).

${ }^{11}$ V. I. Arkhipenko, S. M. Zgirovksiǐ, A. A. Kirillov, and L. V. Simonchik, Plasma Phys. Rep. 28, 858 (2002).

${ }^{12}$ O. Motret, C. Hibert, S. Pellerin, and J. M. Pouvesle, J. Phys. D 33, 1493 (2000).

${ }^{13}$ K. L. Bell, A. Dalgarno, and A. E. Kingston, J. Phys. B 1, 18 (1968). 